\title{
The psychological implications of sleep apnea
}

\author{
Ashish Sarangi MD, E.L. Domingo-Johnson BSN, RN, Lance Mwangi BS, \\ Arham Siddiqui BS, Chia Hsu BS
}

\begin{abstract}
Obstructive sleep apnea (OSA) is a common sleep disorder characterized by collapse or obstruction of the airway with associated hypoxemia. Physiological conditions associated with OSA include hypertension and cardiac arrhythmias; however, OSA is also linked to psychological illnesses and disorders. This study focuses on the relation between OSA and psychological disorders in children and adults by reviewing pertinent literature. The review was conducted using PubMed, which yielded 56 articles between 2015 and 2020. Primary findings included links of OSA to neurological deficiencies, such as decreases in visuospatial ability, attention, and memory, as well as structural defects (e.g., edema and gliosis). Studies also showed a bidirectional relationship between OSA and major depressive disorder. A similar finding is observed between OSA and bipolar disorder, which can be aggravated by atypical antipsychotic treatments. It is important to continue to investigate the clinical manifestations of OSA in adult and children populations to prevent, diagnose, and treat related psychological conditions.
\end{abstract}

Keywords: Sleep apnea syndromes, bipolar disorder, major depressive disorder, cognitive impairment

\section{INTRODUCTION}

Obstructive sleep apnea (OSA) affects about 9\% of the general population. It is characterized by collapse of the airway during sleep, and it often causes intermittent hypoxemia and hypercapnia. According to the guidelines by the American Academy of Sleep Medicine (AASM) Task Force, the severity of sleep apnea is determined by the sum of the number of apnea events plus the number of hypopnea events that occur during an hour of sleep, or the apnea-hypopnea index (AHI), as seen on polysomnography. A normal $\mathrm{AHI}$ is fewer than 5 events per hour of sleep. A mild rating of OSA is defined as 5-14 events per hour, moderate as 15-30 events per hour, and severe as

Corresponding author: E.L. Domingo-Johnson Contact Information: EL.Domingo-Johnson@ttuhsc.edu DOI: $10.12746 /$ swrccc.v8i36.763 more than 30 events per hour. Risk factors for OSA include excessive weight, greater neck circumference, advanced age, and narrowed airway. The most common associated symptoms are snoring at night and daytime sleepiness. The condition is most often treated with a continuous positive airway pressure (CPAP) device to maintain the airway and provide oxygenation at night. Other methods of management include weight loss, mechanical devices to avoid the supine position (including recliners and wedge pillows), airway surgery, and oral appliances including mandibular advancement devices, such as SleepPro's Easifit or SomnoMed's SomnoDent Classic. ${ }^{1}$ Obstructive sleep apnea is often associated with multiple medical disorders, such as hypertension, cardiac arrhythmias, impaired memory, metabolic disorders, and polycythemia. Not only does OSA have physiological implications in patients, but it also has psychological associations. Studies have noted a higher prevalence of sleep apnea in patients who have severe 
mental illnesses, such as cognitive impairment, ${ }^{2}$ major depressive disorder, ${ }^{3}$ and bipolar disorder in adults and children. ${ }^{4}$ This review will analyze studies that explain the links between sleep apnea and psychological disorders in adults and children. This study also reviews current theories pertaining to the interactions between the management of psychological disorders and improvements in the symptoms of OSA.

\section{Methods}

A literature search was conducted using PubMed to search for articles between 2015 to 2020, which yielded 56 articles. The search was done using "Sleep Apnea Syndromes/Psychology", "mandibular advancement devices", and "cognitive impairment" as the $\mathrm{MeSH}$ terms AND Humans AND English, with clinical trial, meta-analysis, randomized control trial, and multicenter study as the subtypes [ptyp]. The "last 5 years" was used as the publication date [PDat]. The appropriate articles were then chosen that fit the criteria.

\section{RESULTS}

Fifty-six articles with the relevant search terms were returned. Seven studies were excluded from our analysis due to inadequate $(<10)$ sample size with questionable external validity. Two studies were excluded due to significant drop out rates in the participants, and one study was excluded due to inadequately reported methods and multiple participants being lost to follow up leading to statistically non-significant results. Our final list of studies used for analyses was based on robust studies with a large sample size, meta-reviews, and cohort designs. In particular, this review focused on studies with a bi-directional relationship between OSA and mood disorders, including depression and bipolar disorder.

\section{Discussion}

Sleep deprivation alone can lead to memory impairment. However, sleep apnea has multiple mechanisms that result in further detrimental effects. The intermittent hypoxia associated with sleep apnea can lead to brain atrophy. Intrathoracic pressure swings can cause a decrease in the exchange of cerebral spinal fluid and interstitial fluid. Obstructive sleep apnea is also associated with structural brain changes, such as edema and gliosis. Sleep fragmentation alters neuronal firing patterns, resulting in a decrease in rapid eye movement sleep, fewer spindles, and slower oscillations. ${ }^{4}$ A meta-review that compared COPD, insomnia, and sleep deprivation found that hypoxia/hypercapnia is consistently associated with impairments in attention, memory, executive functions, psychomotor functions, and language ability. ${ }^{6}$ Short term sleep deprivation is also associated with decreased attention and memory. Visuospatial deficits were unique to OSA.

Pan et al. demonstrated a bidirectional temporal association of OSA and depression in a retrospective cohort study of 6,427 patients in Taiwan. Over a 5-year follow-up period, patients with OSA were more likely to be diagnosed with depression than those without OSA. Conversely, patients diagnosed with depression were more likely to be diagnosed with OSA than patients without OSA $(\mathrm{HR}=2.30) .{ }^{7}$ Currently there are two theories on the correlation between OSA and major depressive disorder. One theory suggests that there is a unidirectional causal relationship because poor sleep quality can easily affect mood and mental health. The other suggests that there could be a common pathophysiology via increased pro-inflammatory cytokines causing neural injury or abnormalities in serotonin uptake affecting both depression and upper airway dilator activity. ${ }^{8}$

Obstructive sleep apnea has been also linked to bipolar disorder; however, the relationship is not well defined. Some literature suggests that the treatment for bipolar disorder may lead to development of sleep apnea. First line treatments for bipolar disorder include lithium and valproic acid supplemented by the use of atypical antipsychotics (e.g., aripiprazole). These drugs are associated with multiple side effects, especially weight gain, which predisposes patients to develop OSA. In addition, a common pharmacologic feature of the atypical antipsychotics is blockade of D2 and 5-HT2A receptors. Previous studies have also shown that serotonergic agonists positively regulate central respiratory drive and increase airway diameter. Therefore, inhibition of this serotonin pathway can 
potentially worsen the symptoms of OSA by increasing upper airway resistance and lowering central respiratory drive. ${ }^{9}$

Furthermore, bipolar disorder is characterized by disrupted sleep-wake schedules, daily activities, and appetite, all of which are integrally related to the patient's circadian system. Mood exacerbations can also be provoked by seasonal patterns and alterations in light intensity. This may explain concomitant irregular control of sleep, hormone levels, and body temperature, all under circadian control. ${ }^{9}$ Thus, whether OSA or bipolar disorder is diagnosed first, each can have a detrimental synergistic effect on the patient if not properly treated.

\section{Conclusions}

Obstructive sleep apnea is a multifaceted disorder that manifests itself not only physiologically but also psychologically. It is therefore necessary for a physician to consider a patient's risk for psychiatric disorders, including major depressive disorder and bipolar disorder. The link between OSA and major depressive disorder and bipolar disorder is not well defined, with only a handful of studies on this relationship. Nonetheless, a clear understanding of OSA's association with psychological disorders can introduce novel methods for its diagnosis, prevention, and treatment in patients.

Article citation: Sarangi A, Domingo-Johnson EL, Mwangi L, Siddiqui SA, Hsu C. The psychological implications of sleep apnea. The Southwest Respiratory and Critical Care Chronicles 2020;8(36):55-57

From: School of Medicine (ELDJ, LM, AS, CH) and Department of Psychiatry(AS), Texas Tech University Health Sciences Center, Lubbock, Texas

Submitted: 5/14/2020

Accepted: $10 / 2 / 2020$

Reviewer: Gilbert Berdine MD

Conflicts of interest: none

This work is licensed under a Creative Commons

Attribution-ShareAlike 4.0 International License.

\section{REFERENCES}

1. Basyuni S, Barabas M, Quinnell T. An update on mandibular advancement devices for the treatment of obstructive sleep apnoea hypopnoea syndrome. J Thorac Dis 2018;10(Suppl 1): S48-S56. doi:10.21037/jtd.2017.12.18

2. Bilyukov RG, Nikolov MS, Pencheva VP, et al. Cognitive impairment and affective disorders in patients with obstructive sleep apnea syndrome. Front Psychiatry 2018;9:357. Published 2018 Aug 7. doi:10.3389/fpsyt.2018.00357

3. Stubbs B, Vancampfort D, Veronese N, et al. The prevalence and predictors of obstructive sleep apnea in major depressive disorder, bipolar disorder and schizophrenia: A systematic review and meta-analysis. J Affective Dis. https://www. sciencedirect.com/science/article/pii/S0165032715311939. Published March 9, 2016. Accessed March 16, 2020.

4. Hodges EL, Marcus CY, Kim JY, et al. Depressive symptomatology in school-aged children with obstructive sleep apnea syndrome: incidence, demographic factors, and changes following a randomized controlled trial of adenotonsillectomy. https://www.ncbi.nlm.nih.gov/pubmed/30212861. Published September 13, 2018. Accessed March 16, 2020.

5. Ahuja S, Chen RK, Kam K, et al. Role of normal sleep and sleep apnea in human memory processing. Nat Sci Sleep 2018; 10:255-269.

6. Olaithe M, Bucks RS, Hillman DR, et al. Cognitive deficits in obstructive sleep apnea: Insights from a meta-review and comparison with deficits observed in COPD, insomnia, and sleep deprivation. Sleep Med Rev 2018;38:39-49.

7. Pan M-L, Hsiao-Mei T, Chien-Chi H, et al. Bidirectional association between obstructive sleep apnea and depression. Medicine 2016;95:e4833.

8. $\mathrm{Xu}$ J, Pang KP, Rotenberg B. Should patients with obstructive sleep apnea be screened for depression? Laryngoscope 2019;129(8):1729-1730.

9. Moonga SS, Mischoulon D, Winkelman JW, et al. Obstructive sleep apnea as a complication of bipolar disorder and its treatment: a review and approach to management. Prim Care Companion CNS Disorders. 2017;19(6):17f02194. Published 2017 Nov 30. doi:10.4088/PCC.17f02194 